\title{
Valorisation of lignin by depolymerisation and fractionation using supercritical fluids and conventional solvents
}

\author{
Eduardo Pérez ${ }^{*}$, Christopher O. Tuck and Martyn Poliakoff
}

The School of Chemistry, University of Nottingham, Nottingham NG7 2RD, UK. eperezve@hotmail.com

\begin{abstract}
A procedure for Lignosulphonate valorisation is investigated. An attempt has been made to obtain diverse value-added products maximizing the atom economy. This procedure is carried in sequential steps starting with an oxidative depolymerization in supercritical water. Next, the reaction mixture is fractionated according to its solubility in water and in ethyl acetate. Several analytical methods - CHN elemental analysis, aqueous GPC and ${ }^{31} \mathrm{P}-\mathrm{NMR}$ - were used to determine the composition of these fractions and to assess their suitability for different applications. Water-insoluble fractions were converted to a ligninderived hydrochar for the synthesis of active carbon of superior quality. Monomers were recovered from bio-oil fraction by supercritical carbon dioxide extraction and the remaining oil is proposed as a potential starting material for the synthesis of polyurethane foams.
\end{abstract}

\section{Keywords}

lignin valorization, fractionation, supercritical water oxidation, supercritical extraction

\section{Abbreviations $^{1}$}

\section{1.-Introduction}

Replacement of fossil sources of carbon for fuels and chemicals by renewable feedstocks is one of the main targets for the development of a sustainable economy. In that regard, the concept of biorefinery, as a structure able to efficiently convert different types of biomass into useful chemicals is envisaged as one promising approach to achieve this target. $[1,2]$

Among all the types of biomass, lignocellulose is the most widely available source of carbon. Lignocellulosic biomass is mainly composed of cellulose, hemicellulose and lignin. Unlike the two first components, which have been successfully converted into a wide range of platform chemicals and bio-fuels, [1] lignin has been relatively

\footnotetext{
${ }^{1}$ GPC: Gel Permeation Chromatography

PH: Preheater

BPR: Back Pressure Regulator

LS: Lignosulphonate

Q: Quench

AcEt: Ethyl Acetate

VAN: Vanillin

GUA: Guaiacol

$\mathrm{AcV}$ : Acetovanillone
} 
underutilized, mainly due to the technical difficulties to depolymerize it. However, to develop a biorefining process that is both cost-efficient and green, lignin must also be processed and valorised. [2]

Many attempts have been made to convert lignin into value-added compounds, most of them via depolymerisation. [2-8] However, a high selectivity and yield towards one specific product has rarely been achieved. Instead a wide range of compounds such as monomers, oligomers, tar, char and gases are obtained. The only notable commercial process has been the production of vanillin from lignosulfonates (LS) $[9,10]$ but this route is less and less competitive in comparison to the petrochemical-based vanillin due to the large amount of wastes generated. [11]

Instead of targeting one single chemical, a more realistic approach would be to convert lignin into a mixture of compounds that together have a particular application such as biofuels, [12,13] adhesives [14] or feedstock for the synthesis of other chemicals.[15,16] Another potentially attractive way forward is to utilize lignin and lignin-derived materials for the synthesis of polymers such as polyurethanes [17-20] acrylics [21] or epoxy resins. [22] However, these starting materials are often unsuitable for reasons such as the presence of impurities (sugars and mineral ashes in kraft lignins and lignosulfonates), high polydispersity and molecular weight or variability in the composition.

One of the main difficulties faced when processing lignin is the complexity of the analysis and the lack of standardized protocols. [20] The structure of lignin is highly variable and it greatly depends on the type of wood and the method of separation from cellulose and hemicellulose. [2] Also, as already mentioned, lignin depolymerization yields a wide range of components and to analyse them one-by-one is not a realistic option. In a previous report, [23] we suggested that measuring overall parameters (such as characteristic chemical functionalities, molecular weight distribution, etc.) can be a costeffective alternative to individual analysis and almost as informative, enabling the choice of an appropriate analytical techniques giving the best relevant information for the particular application targeted.

Most reports about lignin valorisation have focused on obtaining a product (pure or mixture of compounds) to fit a single purpose and the by-products from these treatments would have, in principle, little use and are considered as a residue. However, according to the "atom economy" principle of Green Chemistry, [24] all the matter obtained from lignin processing should be valorised. [2] For instance, Borges da Silva et al. [17] proposed that the by-products obtained from the production of vanillin from lignin could be used for the production of polyurethanes. However, further reports that exploit this idea are still scarce.

In this paper, we want to highlight the importance of an appropriate analytical protocol to design a multi-purpose procedure to valorise lignin. Optimization and analysis must be carried out hand to hand since the results obtained can be used to locate the optimal conditions. Lignosulphonate was depolymerized via oxidative treatment in supercritical water (SCW) and the product fractionated. The different fractions were analysed using CHN elemental analysis, Gel Permeation Chromatography (GPC) and ${ }^{31} \mathrm{P}-\mathrm{NMR}$ to identify their potential application, e.g. active carbon, polymer synthesis, etc. The fractions were further treated to better match their characteristics to those required for a 
particular application. For instance, the bio-oil obtained was extracted with supercritical $\mathrm{CO}_{2}$ to separate monomers from oligomeric compounds. The results of these procedures have been monitored using suitable analytical techniques.

\section{2.- Experimental}

Lignosulphonate, LS (Borregaard, Lignotech) was depolymerized in a continuous flow reactor. Figure 1a shows a simplified diagram of the experimental setup arrangement. An aqueous solution of $\mathrm{H}_{2} \mathrm{O}_{2}(0.811 \% \mathrm{w} / \mathrm{w})$ is pumped through a pre-heater $(\mathrm{PH})$ at ca 400 ${ }^{\circ} \mathrm{C}$ where it decomposes to molecular oxygen. Then the mixture of $\mathrm{O}_{2} / \mathrm{SCW}$ is driven into the reactor where it encounters another aqueous stream (solution of LS, $50000 \mathrm{ppm}$ and $\mathrm{NaOH} 1 \mathrm{M}$ ) flowing in the opposite direction. The reactive mixture is quenched with a third stream of cold water $(\mathrm{Q})$, again flowing in opposed direction. The reaction mixture is cooled down further by a heat exchanger and then depressurized by a Back Pressure Regulator (BPR) located downstream. With this reactor design, residence times of few seconds can be achieved. Reaction temperature and pressure are $390{ }^{\circ} \mathrm{C}$ and $230 \mathrm{bar}$ respectively, as they were the optimum parameters from previous reports. [23] All the components were pumped by means of HPLC pumps. Flowrates are $\mathrm{SCW} / \mathrm{O}_{2}: 15.0$ $\mathrm{mL} / \mathrm{min}$; LS/cat: $5.1 \mathrm{~mL} / \mathrm{min}$, Quench: $7.5 \mathrm{~mL} / \mathrm{min}$. Details of this apparatus can be found elsewhere. $[23,25,26]$ If properly designed, the uncertainty for this experimental layout can be kept below 3\%. [27]

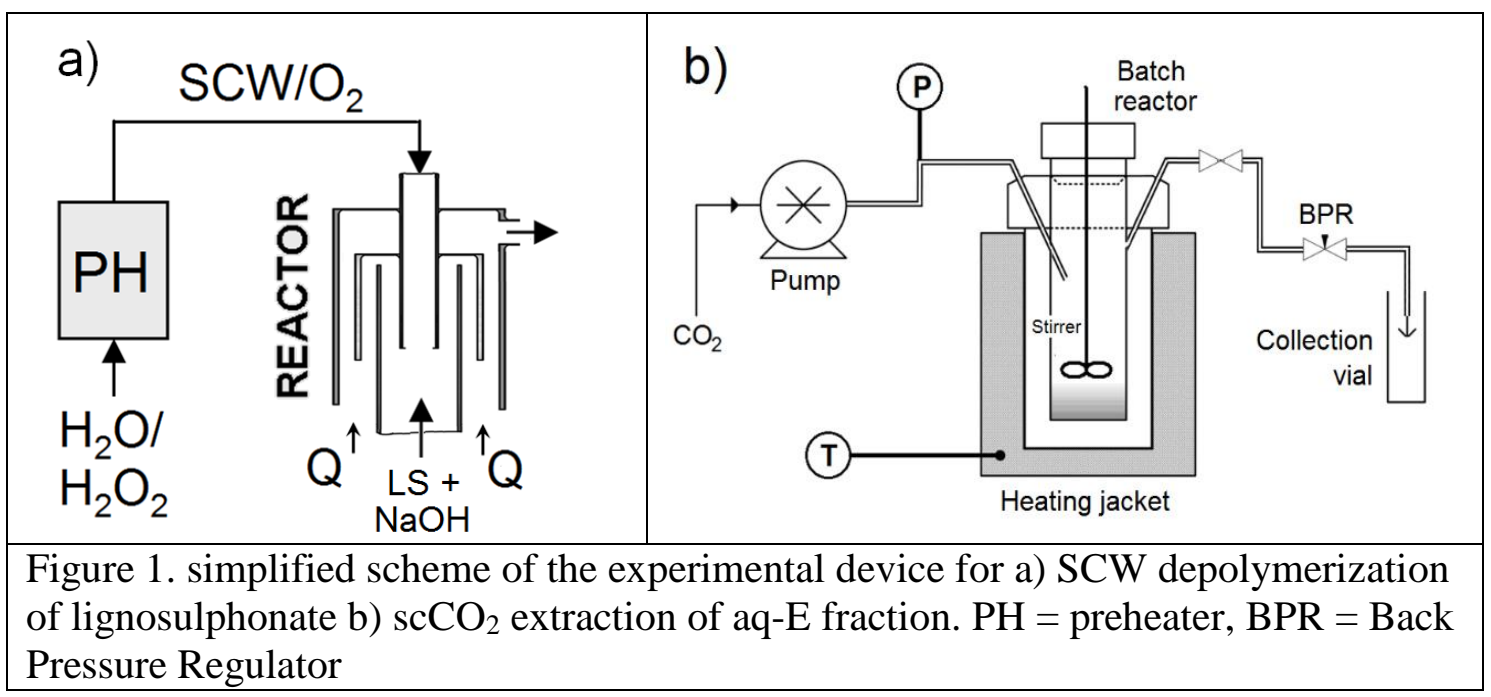

$445 \mathrm{~g}$ of lignosulphonate, was processed in this reactor in a long reaction that lasted several hours. All the samples obtained were combined and the resulting product was fractionated according to its solubility in water and in ethyl acetate following a similar procedure to that previously described [23] The sample was acidified to $\mathrm{pH}=3$ using concentrated sulphuric acid added dropwise A solid fraction precipitates and the watersolubles and water-insolubles are separated by filtration. Both fractions were then extracted with ethyl acetate (AcEt) to obtain an extract and a residue of the aqueous fraction (aq-E and aq- $\mathrm{R}$ respectively), and the extract and the residue of the solid fraction ( $\mathrm{s}-\mathrm{E}$ and $\mathrm{s}-\mathrm{R}$ respectively). The solvent was then removed from each fraction under reduced pressure. An scheme of the fractionation procedure is shown in figure 2 . The main difference between this process and the one that was described in the literature is 
that the water-insolubles are still wet when they are extracted so s-E and s-R could include small amounts of salts from aq-E.

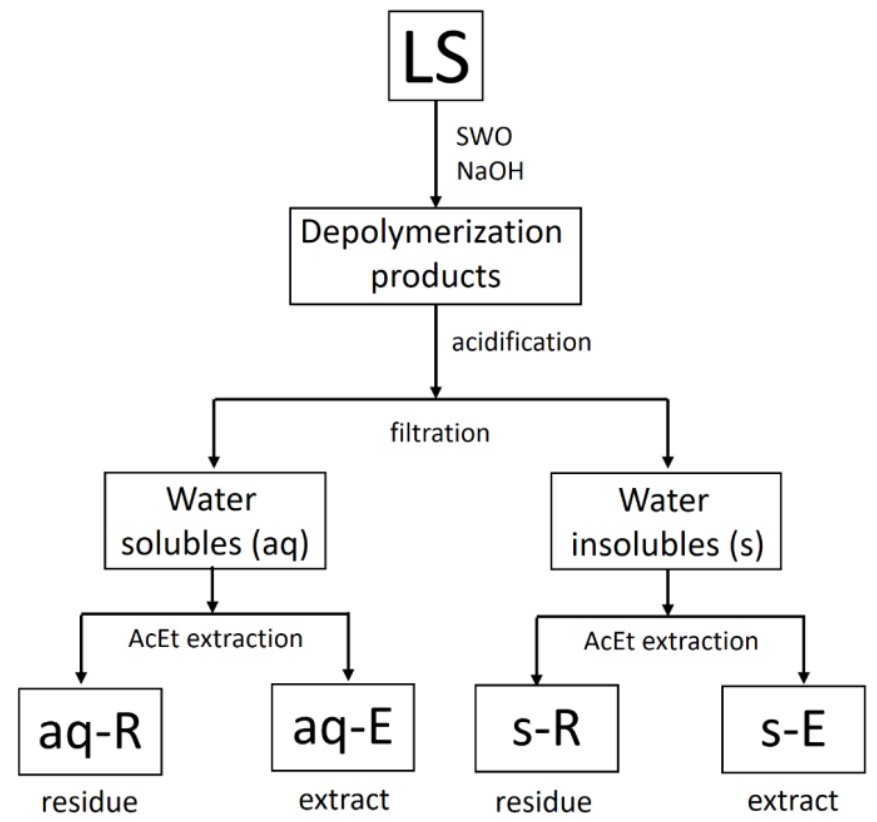

Figure 2. Scheme of the fractionation procedure

Supercritical $\mathrm{CO}_{2}, \mathrm{scCO}_{2}$, extraction was carried out in semi-continuous mode. A schematic of the apparatus is shown in Figure 1b. The sample was placed inside a highpressure reactor of $60 \mathrm{~mL}$ fitted with inlet and outlet tubes. The cell is mechanically stirred and heated up with a resistance. An HPLC pump with cooled pumphead $\left(-10^{\circ} \mathrm{C}\right)$ drove liquid $\mathrm{CO}_{2}$ into the cell while an automated Back Pressure Regulator (BPR) kept the pressure of the system constant. Extracts were recovered after the BPR in a collection vial. Conditions of the extraction were $\mathrm{T}=40^{\circ} \mathrm{C}$ and $\mathrm{P}=150 \mathrm{bar}$. The extraction was carried out in two stages: 1) Batch mode: cell is filled with $\mathrm{CO}_{2}$ at the operating conditions and left for $30 \mathrm{~min}$; 2) flow mode: $1 \mathrm{~g} / \mathrm{min}$ of $\mathrm{CO}_{2}$ for 2 hours.

The fractions from the $\mathrm{HTW}$ depolymerization and from $\mathrm{scCO}_{2}$ extraction were exhaustively analysed following the protocols described in reference 23. A summary of these procedures is given next:

Elemental microanalyses for $\mathrm{C}, \mathrm{H}$, and $\mathrm{N}$ were carried out on an Exeter Analytical CE440 elemental analyser.

Functionality, $\mathrm{F}_{\mathrm{OH}}$, was defined as the average number of -OH groups per 7 atoms of carbon (guaiacyl unit). It was determined by ${ }^{31} \mathrm{P}-\mathrm{NMR}$ after derivatization with 2-Chloro4,4,5,5-tetramethyl-1,3,2-dioxaphospholane. [28] This technique distinguishes between aromatic, aliphatic - $\mathrm{OH}$ and $-\mathrm{COOH}$ moieties. An internal standard (cyclohexanol) was added to determine the concentration of each type of hydroxyl and the functionality.

High performance liquid chromatography (HPLC) was used to quantify the yields of the monomers obtained from lignin depolymerisation. The analysis was performed using an AgilentTM 1100 series with an XterraTM C-18 column and a single wavelength UV detector at $230 \mathrm{~nm}$. 
Molecular weight distribution was obtained by Gel Permeation Chromatography (GPC) using a modified AgilentTM 1100 HPLC fitted with a GPC column (Jordi Gel Sulphonated Plus $10000 \AA 250$ x $10 \mathrm{~mm})$. An isocratic method $(1 \mathrm{~mL} / \mathrm{min})$ is followed using a mixture of water + methanol 90:10 (v/v) adjusted to $\mathrm{pH}=12$ as the mobile phase. The temperature of the column was $35^{\circ} \mathrm{C}$ and the injection volume is $25 \mu \mathrm{L}$. Two detectors, Refractive Index and UV-Vis $(254 \mathrm{~nm})$ were placed in series with a delay in retention time of $0.31 \mathrm{~min}$.

\section{3.-Results and discussion}

\section{1.- Assigning potential uses to the fractions (Mass balance and analysis).}

Table 1 summarizes the main characteristic parameters of the different products obtained from SCW depolymerization of lignin and subsequent fractionation. $24.7 \%$ of the starting lignin was recovered in the aq-E, s-E and s-R fractions. The rest of the mass has been either remained unreacted, was lost during the fractionation procedure or has been totally oxidized to $\mathrm{CO}_{2}$. Also, starting LS has an inorganic content of ca 20\% [29] that has probably been concentrated in the aq- $\mathrm{R}$ fraction. [23]

Table 1. Yield, $\mathrm{CHN}$ elemental analysis and $-\mathrm{OH}$ functionality for the main fractions obtained from SCW oxidative depolymerization of LS.

\begin{tabular}{|l|l|l|l|l|}
\hline & LS & aq-E & s-E & s-R \\
\hline \% Yield & - & 14.9 & 7.7 & 2.1 \\
\hline \multicolumn{5}{|l|}{ Elemental Analysis } \\
\hline \%C & 38.45 & 58.3 & 66.9 & 55.5 \\
\hline \%H & 4.61 & 5.63 & 5.10 & 3.94 \\
\hline \%Other & 56.94 & 36.0 & 28.0 & 40.6 \\
\hline O:C (mol/mol) & 0.721 & 0.463 & 0.314 & 0.549 \\
\hline H:C (mol/mol) & 1.44 & 1.16 & 0.91 & 0.85 \\
\hline Functionality, FoH & - & 0.752 & 0.356 & 0.321 \\
\hline Aromatic -OH & - & 0.135 & 0.031 & 0.068 \\
\hline Aliphatic $-\mathrm{OH}$ & - & 0.353 & 0.079 & 0.093 \\
\hline COOH & - & 1.28 & 0.47 & 0.50 \\
\hline TOTAL & - & -
\end{tabular}

Values of CHN elemental analysis are displayed in Table 1. Negligible amounts of nitrogen were detected in all of the fractions. The "\%Other" figure corresponds to the mass of the sample not accounted for $\mathrm{C}$ and $\mathrm{H}$. i.e. oxygen and possibly inorganic ash but the latter is not expected to be significant in the fractions analysed. [23] $\mathrm{H}: \mathrm{C}$ is the molar ratio of hydrogen and carbon in the sample. It gives some indication of the relative proportions of double bonds; cycles and aromatic rings vs aliphatic material present. Ratio H:C varies in the following order: aq- $\mathrm{R}>\mathrm{s}-\mathrm{E}>\mathrm{s}-\mathrm{R}$. Which indicates a higher proportion of aromatic rings/double bounds in the solid fractions. This is probable because the solids are products from repolymerization by condensation reactions. [23]

The so-called Krevelen diagrams [30] depict \%C, \% $\mathrm{H}$ and $\% \mathrm{O}$ as $\mathrm{H}: \mathrm{C}$ vs O:C atomic ratios. They usually reveal some interesting information about the properties of the 
material and the transformations carried out. [31] In this case, these diagrams can be built making some assumptions: $\% \mathrm{O}$ can be estimated as the difference between the $\%$ Other and \%ash in the feedstocks.[32] \%ash for LS and aq-R is $20 \%$ and $0 \%$ respectively [29]. Assuming the inorganic ash comes from sodium sulfonate groups present in LS and since no \%S is present in the rest of the fractions (s-R, aq-E), a plausible approximation is to consider that these former fractions contain no ash. Figure 3 shows estimated Krevelen diagrams for the samples in Table 1 and some others obtained from reference 23 for comparison.

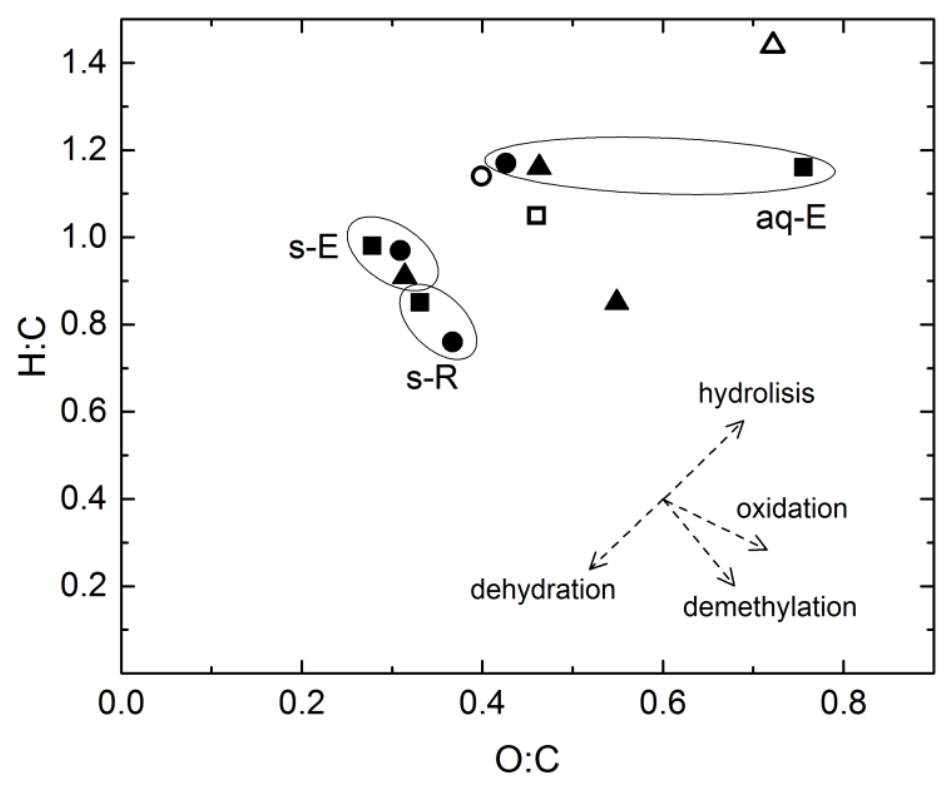

Figure 3. Estimated van Krevelen diagram for different lignins and fractions from their oxidative depolymerization. $(\triangle, \mathbf{A})$ : LS, This work, $(\square, \boldsymbol{\square})$ : LS, reference $23(\bigcirc, \boldsymbol{O})$ Organosolv, reference 23. Hollow symbols correspond to the starting lignins. The arrows indicate the trajectory of different kind of transformations.[31] Hydrolisis and dehydration follow lines of slope +2 (but opposite direction). Slope of demethylation and oxidation lines depends of the reference point which was arbitrarily chosen as (0.4, 1). then demethylation line has a slope of -2.5 and oxidation line of -0.625 .

Figure 3 shows some interesting information that is plausible with previously reported data.[23] All samples S-R and s-E are grouped in two differentiated clusters which indicates that they have similar chemical composition regardless the original lignin and the treatment. These fractions are typical of hydrochar materials $[33,34]$ and seem to have been originated from dehydration reactions. [33] The exception is s-R in this work which lies at higher values of $\mathrm{O}: \mathrm{C}$, suggesting the presence of inorganic impurities as stated below. Notice also that s-R cluster is displaced to higher O:C and lower H:C values which indicates less number of methyl groups as confirmed by NMR in reference 23.

Aq-E have a more variable chemical composition. In general, they are located at higher values of $\mathrm{H}: \mathrm{C}$ and $\mathrm{O}: \mathrm{H}$ than the starting lignin indicating it undergoes a depolymerization via hydrolysis. However, the slope is much lower than +2 which is consistent to additional oxidation mechanisms. Initial lignins lie within typical values. 
[33] The exception is the LS used in this work, which has unusually higher values for $\mathrm{H}: \mathrm{C}$ and $\mathrm{O}: \mathrm{C}$. This can be indicative of leftovers of carbohydrates or cellulose in the lignin,[34] which would also explain the unusually low yields as these would burn in oxidative environment.

Table 1 also shows the proportion of $-\mathrm{OH}$ moieties and the total functionality calculated from ${ }^{31} \mathrm{P}-\mathrm{NMR}$. Aq-E fraction shows a particularly high functionality with $1.28-\mathrm{OH}$ groups per $\mathrm{C} 7$ unit. $\mathrm{FOH}_{\mathrm{OH}}$ for the solid fractions is much lower. The proportion of $-\mathrm{COOH}$ moieties is relatively high for all the fractions due to oxidative ring opening via quinone intermediates.[23]

Figure 4 shows the GPC chromatograms for the three main fractions and the initial LS. The peaks between 11.6 and 12.5 min correspond to monomers from depolymerization; retention times between 11 and $11.6 \mathrm{~min}$ are typical of oligomeric compounds (dimers and trimers, $\mathrm{M}=250-1000 \mathrm{D}$ aprox.) whereas peaks at lower retention times correspond to higher molecular weight fractions.[23]

The solid fractions were mainly composed of high molecular weight compounds and a small amount of oligomers (particularly in s-E). This is consistent with what was obtained previously. [23] However, aq-E shows three peaks, the two larger ones correspond to monomeric and oligomeric components; a much smaller peak at retention time between 11 and 10.5 indicates higher molecular weight compounds.[23]

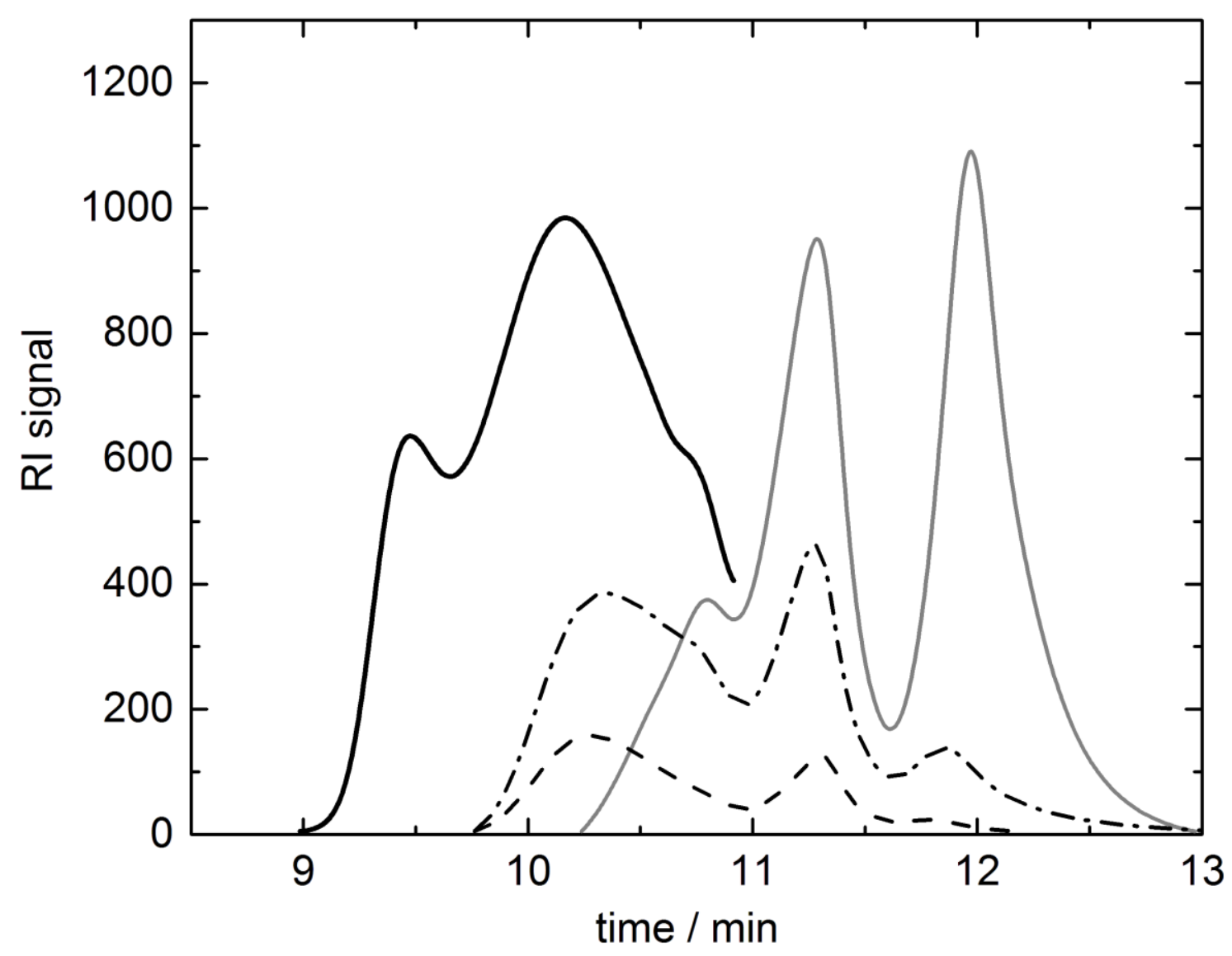

Figure 4. GPC traces (molecular weight distribution) for ( - ): $\mathrm{LS},(-)$ : aq-E fraction, (-----): s-E fraction, (---): s-R fraction. 
From the previous analyses, one can observe two distinctive types of fractions. On one hand, aq-E shows a high degree of depolymerisation, and aromatic functionality, and the molecular weight distribution determined by GPC reveals that this fraction contains most of the monomers generated by depolymerisation. Having the monomers concentrated into one fraction is desirable because it makes it easier and more efficient to recover them. Once separated, the remaining oligomeric mixture could be used as a raw material for polyurethane synthesis.

s-E and s-R on the other hand contains repolymerization products with a high content in carbon, high aromaticity and lower functionality. [23] These last fractions, which are usually envisaged and undesirable by-products are the so-called hydrochar with potential uses in synthesis of carbon materials, among others. [35]

\section{2.- Solid fractions as precursors for active carbon synthesis}

Although s-E and s-R have similar characteristics, [23] a closer inspection of Table 1 reveals significant differences in their carbon content. The $\mathrm{H}: \mathrm{C}$ ratio for the s-R is the lowest of all the fractions indicating high aromaticity but the figure for \%Other is anomalously high. Krevelen diagram in figure 3 also indicates an inconsistent composition for this particular sample. This suggests that this fraction could contain a mineral impurity, which is possible because the water insoluble solids are not dried before extraction and therefore they must contain a certain amount of $\mathrm{Na}_{2} \mathrm{SO}_{4}$ from neutralization that is concentrated in aq-R.

s-E has the appearance of a very viscous dark tar. It also contains a certain amount of low molecular weight compounds which may give the material its fluid consistency which makes it difficult to handle. The tar was heated in an oven at $80^{\circ} \mathrm{C}$ for three days which turned it into a solid material that could not be redissolved in EtAc. GPC was used to monitor the progress of this treatment (see figure 5 which was measured on a similar fraction). Upon thermal treatment, a chemical change occurs to the sample; the proportion of low molecular weight components (oligomers) decreases while the high molecular weight components increase.

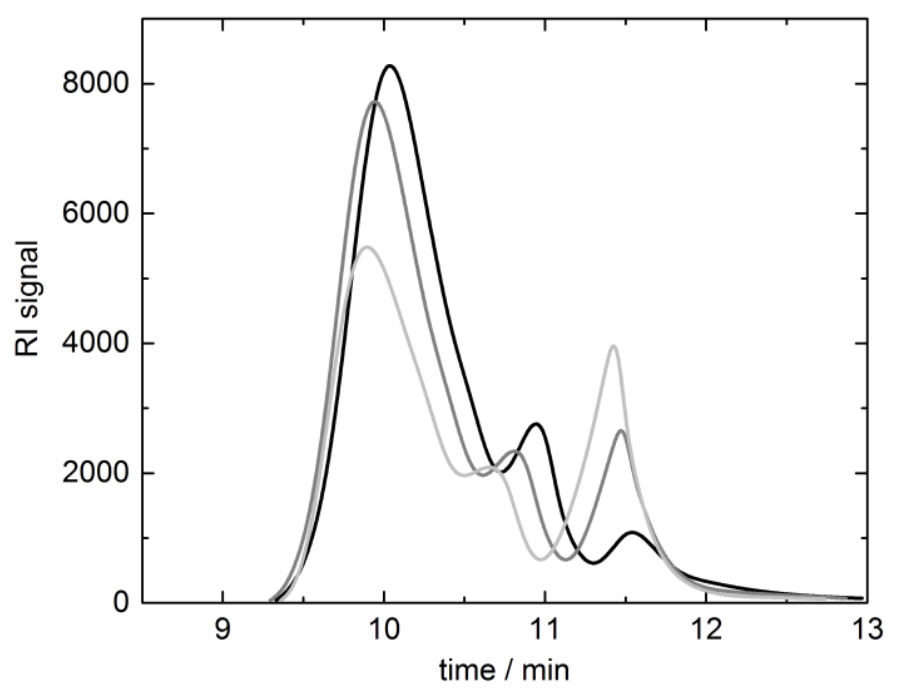

Figure 5. Effect of thermal treatment on molecular weight distribution for an aq-E fraction. $\mathrm{Tb}=80^{\circ} \mathrm{C}$ after: ( - ): 0 days, $(-)$ : 1 day, $(-)$ ): 3 days. 
The resulting solid (Lignin Derived Hydrochar) was used for further investigation by Sangchoom and Mokaya. [29] They demonstrated that it was an excellent precursor for the synthesis of active carbon, since it did not require further demineralization steps and has a high carbon content. Certainly, the inorganic contents, i.e. the sulphate from LS and from neutralization of $\mathrm{NaOH}$ were retained in other fractions as described above. Moreover, the resulting active carbons had superior properties in terms of surface area and gas uptake. [29]

\section{3.- Recovery of the monomers from the liquid fraction.}

Many separation techniques have been applied to isolate the monomers from depolymerization reaction mixtures. [36] Liquid extraction using a range of organic solvents is one of the preferred strategies because of its simplicity. In this work, we chose ethyl acetate as the extraction solvent due to its low toxicity. Supercritical fluid extraction (SFE) has also been applied to isolate vanillin directly from the solution but we preferred the approach of Klemola and Tuovinen [37] where they performed a sequential extraction first with toluene and then with $\mathrm{scCO}_{2}$. In our work, the bio-oil fraction (aq-E) rich in monomers was extracted using $\mathrm{scCO}_{2}$ and both the extract and residue were analysed to understand the extraction process and to establish whether further uses for the materials obtained. The $\mathrm{scCO}_{2}$ extraction was also compared to an ordinary extraction with toluene.

A one-step liquid extraction with toluene was done as follows: $0.5 \mathrm{~g}$ of aq-E and $25 \mathrm{~mL}$ of toluene were placed in a capped flask. The mixture was stirred for $48 \mathrm{~h}$. the extract was recovered and the solvent was removed in a rotary evaporator. The supercritical $\mathrm{CO}_{2}$ extraction to $1.6 \mathrm{~g}$ of aq-E was carried out using the equipment and procedure described in the experimental section.

The yield of extract was calculated by mass in relation to the mass of the original sample of aq-E. Using toluene, $46 \%$ of extract was obtained whereas using $\mathrm{scCO}_{2}$, the proportion of extract was $31 \%$. Figure 6 shows a comparison of the GPC chromatograms for the extract and residues using these solvents. The extracts were mainly composed of monomeric compounds with a small proportion of oligomers. However, comparing the relative size of the peaks, it is clearly seen that $\mathrm{scCO}_{2}$ extraction is much more selective towards monomers than toluene extraction. 


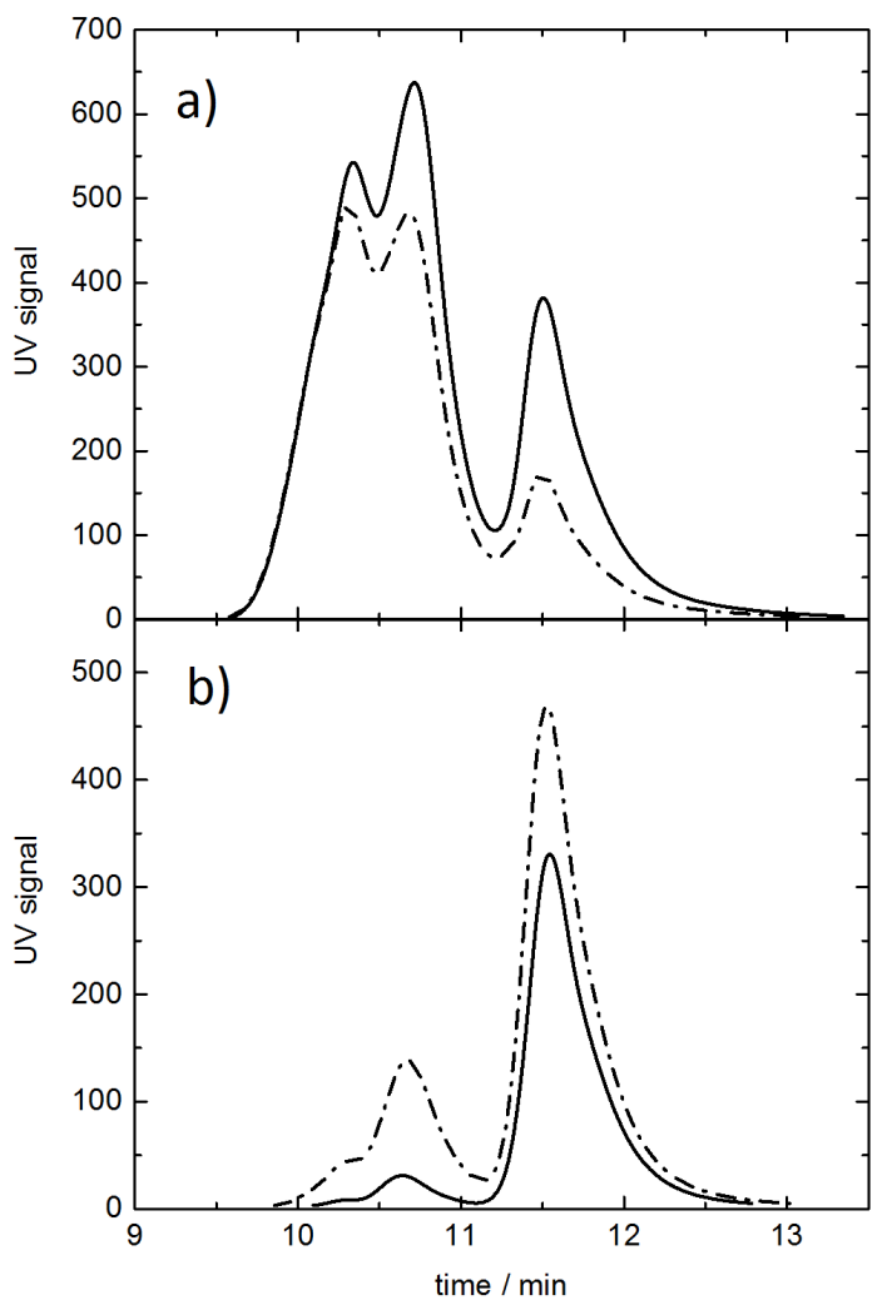

Figure 6. Comparative GPC analysis of the products from the extraction of bio-oil aq-E using: (—): $\mathrm{scCO}_{2},(-\cdot-\cdot-)$ : Toluene. a) residue, b) extract.

The extract and residues from $\mathrm{scCO}_{2}$ extraction of the aq-E fraction were analysed in more detail by HPLC and ${ }^{31}$ P-NMR. Figure 7 Show the HPLC chromatograms for the extract and the residue 


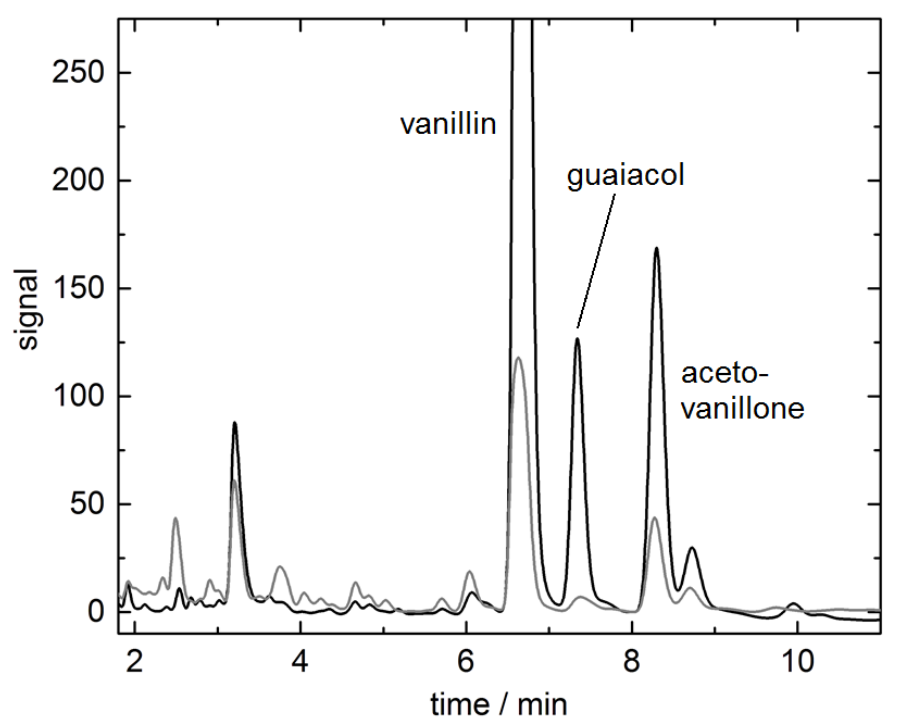

Figure 7. HPLC traces for the products from the extraction of aq-E with $\mathrm{scCO}_{2}$. $(-)$ : extract, ( - ): Residue. Peaks at retention times of 6.6, 7.3 and 8.3 min correspond to Vanillin, Guaiacol and acetovanillone respectively.

Three major peaks were observed in the HPLC trace of the extract: Vanillin, VAN, Guaiacol, GUA and acetovanillone, AcV. The presence of these components was confirmed by GC-MS. The concentration of the main components was quantified. The analysis of the residue show that small amounts of VAN and AcV still remained after SFE. GUA however was almost completely extracted (Table 2, first column).

The second column of Table 2 lists the proportion of each monomer referred to the total mass of extract. i.e. the selectivity. Only three compounds account for $57.4 \%$ of the total extract. VAN is concentrated up to $34.5 \%$. Isolating a desired compound would be obviously much easier from this mixture. If the amount of each compound is related to the initial amount of lignin loaded an overall yield of monomers of $2.64 \%$ is obtained, which is in the same order of magnitude as previous reports. [23]

Table 2. Quantification of Vanillin, Guaiacol and acetovanillone extracted from aq-E: percentage extracted, selectivity and overall yield.

\begin{tabular}{|l|l|l|l|}
\hline & $\%$ Extracted & $\begin{array}{l}\%(w / w) \\
\text { Selectivity }\end{array}$ & $\begin{array}{l}\% \text { Yield } \\
\text { (overall) }\end{array}$ \\
\hline VAN & $87 \%$ & 34.5 & 1.59 \\
\hline GUA & $92 \%$ & 16.3 & 0.75 \\
\hline AcV & $78 \%$ & 6.5 & 0.30 \\
\hline TOTAL & & 57.4 & 2.64 \\
\hline
\end{tabular}

In order to optimize a procedure based on $\mathrm{CO}_{2} \mathrm{SFE}$ of the bio-oil, It would be interesting to investigate if $\mathrm{scCO}_{2}$ extracts preferentially one type of compounds. In that regard analysis of functionalities for the extract and residue can be useful. In figure 8, the proportion of $-\mathrm{OH}$ moieties (aliphatic, aromatic and $-\mathrm{COOH}$ ) determined by ${ }^{31} \mathrm{P}$ NMR are shown. Extraction with $\mathrm{scCO}_{2}$ is selective towards the aromatic (guaiacyl) units in contrast to molecules containing carboxylic groups, which tend to remain in the residue. The proportion of aliphatic groups, remains almost unaltered upon extraction. 


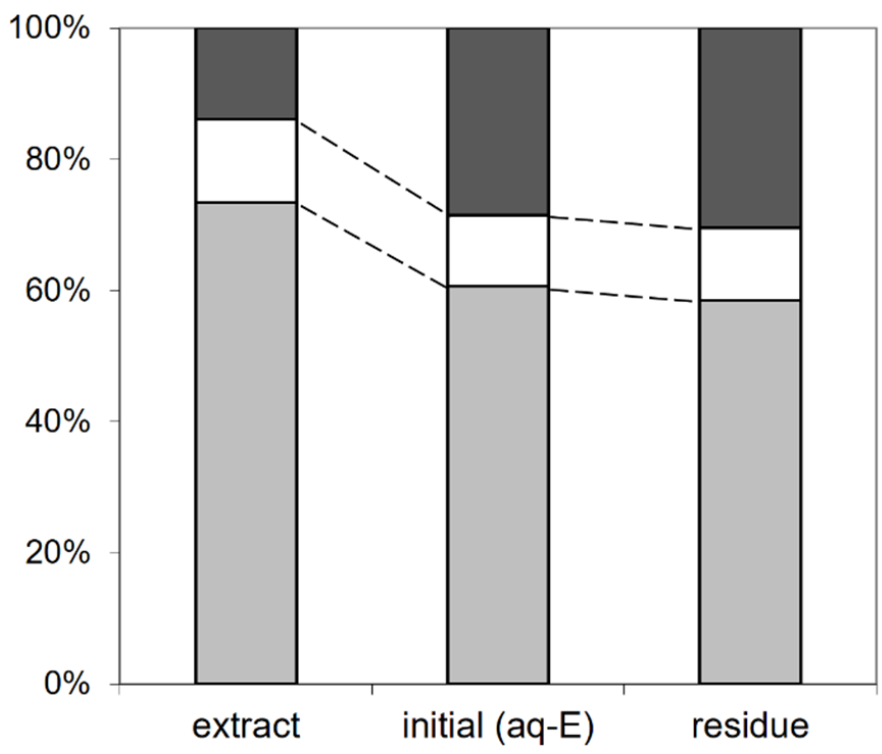

Figure 8. Proportion of the different types of $-\mathrm{OH}$ moieties distributed over the extract and the residue after $\mathrm{scCO}_{2}$ extraction. ( $\square$ ): Carboxylic, $(\square)$ : Aliphatic, ( $\square$ ): Aromatic.

A final functionality of 0.887 hydroxyl groups per $\mathrm{C} 7$ unit is determined for the residue obtained from $\mathrm{scCO}_{2}$ extraction. That is equivalent to $6.16 \mathrm{~mol}_{\mathrm{OH}} / \mathrm{g}$ or to a hydroxyl index of 345. Optimum values for a material to be used for the synthesis of a polyurethane foam are between 300 and 800. [17] Moreover, the material obtained has a moderate molecular weight (250 - $1000 \mathrm{D}$ approx.) and it is not expected to have mineral ash. [23] These characteristics seem to be suitable for the objective pursued. [18] Further research could be done to upgrade the quality of this material but, as shown, a successful optimization procedure must involve the determination of the functionality and molecular weight.

\section{Conclusions}

A procedure to convert lignosulfonate into several value-added materials by oxidative depolymerization in supercritical water followed by fractionation has been designed. Water-insoluble fractions consist of a high molecular weight material with high carbon content. They were converted to a lignin-derived hydrochar which is an excellent starting material for the synthesis of active carbon of superior quality. Bio-oil fractions are composed by monomers and oligomers. The former are recovered by $\mathrm{scCO}_{2}$ extraction whereas the latter form a material of high functionality and moderate molecular weight suitable for the synthesis of polyurethane foams. Proper analytical methods such as CHN elemental analysis, GPC, HPLC and ${ }^{31} \mathrm{P}-\mathrm{NMR}$ were essential to determine the composition and potential uses of each fraction in each step of the valorisation process.

\section{Acknowledgements}

The authors acknowledge EPRSC, the University of Nottingham for financial support. The authors also want to thank Adrienne Davis, Gill Stephens, Abada Zahra, Peter Fields, Richard Wilson and Mark Guyler for their help. 


\section{Figure captions}

Figure 1. simplified scheme of the experimental device for a) SCW depolymerization of lignosulphonate b) $\mathrm{scCO}_{2}$ extraction of aq-E fraction. $\mathrm{PH}=$ preheater, $\mathrm{BPR}=\mathrm{Back}$ Pressure Regulator

Figure 2. Scheme of the fractionation procedure

Figure 3. Estimated van Krevelen diagram for different lignins and fractions from their oxidative depolymerization. $(\triangle, \mathbf{\Delta})$ : LS, This work, $(\square, \boldsymbol{\square})$ : LS, reference $23(\bigcirc, \boldsymbol{O})$ Organosolv, reference 23. Hollow symbols correspond to the starting lignins. The arrows indicate the trajectory of different kind of transformations.[31] Hydrolisis and dehydration follow lines of slope +2 (but opposite direction). Slope of demethylation and oxidation lines depends of the reference point which was arbitrarily chosen as $(0.4$, 1). then demethylation line has a slope of -2.5 and oxidation line of -0.625 .

Figure 4. GPC traces (molecular weight distribution) for (-): LS, ( - ): aq-E fraction, (-----): s-E fraction, (---): s-R fraction.

Figure 5. Effect of thermal treatment on molecular weight distribution for an aq-E fraction. $\mathrm{Tb}=80^{\circ} \mathrm{C}$ after: ( - ): 0 days, $(-)$ : 1 day, $(-)$ : 3 days.

Figure 6. Comparative GPC analysis of the products from the extraction of bio-oil aq-E using: (—): $\mathrm{scCO}_{2},(-\cdot-\cdot-)$ : Toluene. a) residue, b) extract.

Figure 7. HPLC traces for the products from the extraction of aq-E with $\mathrm{scCO}_{2}$. $(-)$ : extract, (-): Residue. Peaks at retention times of 6.6, 7.3 and 8.3 min correspond to Vanillin, Guaiacol and acetovanillone respectively.

Figure 8. Proportion of the different types of -OH moieties distributed over the extract and the residue after $\mathrm{scCO}_{2}$ extraction. ( $\square$ ): Carboxylic, $(\square)$ : Aliphatic, ( $\square$ ): Aromatic. 
[1] C.O. Tuck, E. Pérez, I.T. Horváth, R.A. Sheldon, M. Poliakoff, Valorization of Biomass: Deriving More Value from Waste, Science, 337 (2012) 695-699.

[2] J. Zakzeski, P.C.A. Bruijnincx, A.L. Jongerius, B.M. Weckhuysen, The Catalytic Valorization of Lignin for the Production of Renewable Chemicals, Chem. Rev., 110 (2010) 3552-3599.

[3] M.P. Pandey, C.S. Kim, Lignin Depolymerization and Conversion: A Review of Thermochemical Methods, Chem. Eng. Technol., 34 (2011) 29-41.

[4] S. Kang, X. Li, J. Fan, J. Chang, Hydrothermal conversion of lignin: A review, Renew. Sust. Energ. Rev., 27 (2013) 546-558.

[5] H. Lange, S. Decina, C. Crestini, Oxidative upgrade of lignin - Recent routes reviewed, Eur. Polym. J., 49 (2013) 1151-1173.

[6] C.A. Gasser, G. Hommes, A. Schaffer, P.F. Corvini, Multi-catalysis reactions: new prospects and challenges of biotechnology to valorize lignin, Appl. Microbiol. Biot., 95 (2012) 1115-1134.

[7] M. Kleinert, T. Barth, Towards a Lignincellulosic Biorefinery: Direct One-Step Conversion of Lignin to Hydrogen-Enriched Biofuel, Energ. Fuel., 22 (2008) 13711379.

[8] G.T. Beckham, C.W. Johnson, E.M. Karp, D. Salvachu, D.R. Vardon, Opportunities and challenges in biological lignin valorization, Curr. Opin. Biotech., 42 (2016) 40-53.

[9] M. Fache, B. Boutevin, S. Caillol, Vanillin Production from Lignin and Its Use as a Renewable Chemical, ACS Sustain. Chem. Eng., 4 (2016) 35-46.

[10] M.B. Hocking, Vanillin: Synthetic Flavoring from Spent Sulfite Liquor, J. Chem. Edu., 74 (1997) 1055-1059.

[11] R.A. Sheldon, H. van Bekkum, Fine Chemicals through Heterogeneous Catalysis, Wiley-VCH Verlag GmbH, Weinheim, Germany, 2000.

[12] M. Kleinert, T. Barth, Towards a Lignincellulosic Biorefinery: Direct One-Step Conversion of Lignin to Hydrogen-Enriched Biofuel, Energ. Fuel., 22 (2008) 13711379.

[13] W.W. Zmierczak, J.D. Miller, Processes for Catalytic Conversion of Lignin to Liquid Bio-Fuels, International Patent WO/2006/119357 (2006).

[14] S. Yang, T.-Q. Yuan, M.-F. Li, R.C. Sun, Hydrothermal degradation of lignin:

Products analysis for phenol formaldehyde adhesive synthesis, Int. J. Biol. Macromol., 72 (2015) 54-62.

[15] A.M. Elfadly, I.F. Zeid, F.Z. Yehia, A.M. Rabie, M.M. Aboualala, S.-E. Park, Highly selective BTX from catalytic fast pyrolysis of lignin over supported mesoporous silica. Int. J. Biol. Macromol., 91, (2016) 278-293.

[16] A.G. Demesa, A. Laari, I. Turunen, M. Sillanpää, Alkaline Partial Wet Oxidation of Lignin for the Production of Carboxylic Acids, Chem. Eng. Technol., 38 (2015) 2270-2278. 
[17] E.A. Borges da Silva, M. Zabkova, J.D. Araújo, C.A. Cateto, M.F. Barreiro, M.N. Belgacem, A.E. Rodrigues, An integrated process to produce vanillin and lignin-based polyurethanes from Kraft lignin, Chem. Eng. Res. Des., 87 (2009) 1276-1292.

[18] D.V. Evtuguin, J.P. Andreolety, A. Gandini, Poliurethane based on oxygenorganosolv lignin, Eur. Polym. J., 34 (1998) 1163-1169.

[19] M. Wang, C. Xu, M. Leitch, Liquefaction of cornstalk in hot-compressed phenolwater medium to phenolic feedstock for the synthesis of phenol-formaldehyde resin, Bioresource Technol., 100 (2009) 2305-2307.

[20] C.A. Cateto, M.F. Barreiro, A.E. Rodrigues, M.C. Brochier-Salon, W. Thielemans, M.N. Belgacem, Lignins as Macromonomers for Polyurethane Synthesis: A Comparative Study on Hydroxyl Group Determination, J. Appl. Polym. Sci., 109 (2008) 3008-3017.

[21] R. Chen, B.V. Kokta, J.L. Valade, Study on the graft copolymerization of lignosulfonate and acrylic monomers, J. Appl. Polym. Sci., 25, (1980) 2211-2220.

[22] M. Fache, B. Boutevin, S. Caillol, Epoxy thermosets from model mixtures of the lignin-to-vanillin process, Green Chem., 18 (2016) 712-725.

[23] E. Pérez, C.O. Tuck, Quantitative analysis of products from lignin depolymerisation in high-temperature water, Fuel Process. Technol. (2017) Submitted.

[24] P.T. Anastas, C.J. Warner, Green Chemistry: Theory and Practice, Oxford University Press, 1998.

[25] C.O. Tuck, Selective Oxidation of Lignin in High Temperature Water, $\mathrm{PhD}$ dissertation, School of Chemistry, The University of Nottingham, 2013.

[26] E. García-Verdugo, E. Vernardou, W.B. Thomas, K. Whiston, W. Partenheimer, P.A. Hamley, M. Poliakoff, Is it Possible to Achieve Highly Selective Oxidations in Supercritical Water? Aerobic Oxidation of Methylaromatic Compounds, Adv. Synth. Catal., 346 (2004) 307-316.

[27] E. Pérez, M.L. Thomas, D. Housley, P.A. Hamley, J. Fraga-Dubreuil, J. Li, E. Lester, M. Poliakoff, Selective aerobic oxidation of para-xylene in sub- and supercritical water. Part 3: effects of geometry and mixing in laboratory scale continuous reactors, RSC Adv. 6 (2016) 11289-11294.

[28] A. Granata, D.S. Argyropoulos, 2-Chloro-4,4,5,5-tetramethyl-1,3,2dioxaphospholane, a Reagent for the Accurate Determination of the Uncondensed and Condensed Phenolic Moieties in Lignins. J. Agric. Food Chem., 43 (1995) 1538-1544.

[29] W. Sangchoom, R. Mokaya, Valorization of Lignin Waste: Carbons from Hydrothermal Carbonization of Renewable Lignin as Superior Sorbents for $\mathrm{CO}_{2}$ and Hydrogen Storage, ACS Sustain. Chem. Eng., 3 (2015) 1658-1667.

[30] D.W. van Krevelen, J. Schuyer, Coal Science, Elsevier, Amsterdam, 1957.

[31] C.L. Heald, J.H. Kroll, J.L. Jimenez, K.S. Docherty, P.F. DeCarlo, A.C. Aiken, Q. Chen, S.T. Martin, D.K. Farmer, P. Artaxo, A simplified description of the evolution of organic aerosol composition in the atmosphere, Geophys. Res. Lett., 37 (2010) L08803

[32] M.M. Mossoba, Spectral Methods in Food Analysis: Instrumentation and Applications, CRC Press, 1998.

[33] D. Licursi, C. Antonetti, J. Bernardini, P. Cinelli, M.B. Coltelli, A. Lazzeri, M. Martinelli, A.M.R. Galletti, Characterization of the Arundo Donax L. solid residue from 
hydrothermal conversion: Comparison with technical lignins and application perspectives, Ind. Crops Prod., 76 (2015) 1008-1024.

[34] S. Schimmelpfennig, B. Glaser, One Step Forward toward Characterization: Some Important Material Properties to Distinguish Biochars, J. Environ. Qual., 41 (2012) 1001-1013.

[35] J.A. Libra, K.S. Ro, C. Kammann, A. Funke, N.D. Berge, Y. Neubauer, M.-M. Titirici, C. Fuhner, O. Bens, J. Kern, K.-H. Emmerich, Hydrothermal carbonization of biomass residuals: A comparative review of the chemistry, processes and applications of wet and dry pyrolysis, Biofuels, 2 (2011) 71-106.

[36] M.I.F. Mota, P.C.R. Pinto, J.M. Loureiro, A.E. Rodrigues. Recovery of Vanillin and Syringaldehyde from Lignin Oxidation: A Review of Separation and Purification Processes, Sep. Purif. Rev., 45 (2016) 227-259.

[37] A. Klemola, J. Tuovinen, Method for the production of vanillin, U.S. Patent 4847422 (1989) 